\title{
Factors Affecting Post Nephrostomy Removal Urinary Leakage after Percutaneous Nephrolithotomy (PCNL)
} Karwan Asaad Othman (MBChB, KBMS) ${ }^{1}$, Shawqi George Gazala (MBChB, MRCS, LRCP, FRCS Ed, FRCS Ld $)^{2}$ and Nihad Rifaat Jawad (MBChB,PhD) ${ }^{3}$

\begin{abstract}
Background: Prolonged urinary leakage after nephrostomy tube removal leads to increased morbidity of PCNL and increased hospitalization.

Objective: To evaluate factors affecting post nephrostomy removal urinary leakage after percutaneous nephrolithotomy.

Patients and Methods: One hundred eight (108) patients older than 18 years who underwent PCNL were studied prospectively. The reasons that can affect urinary leakage postoperatively were divided into 4 categories according to individual factors including age, gender, and body mass index; Renal factors; including, previous operation, history of ESWL, parenchymal cortical thickness, and grades of hydronephrosis; stone factor (stone burden); and surgical factors including the number of tracts, presence of double $\mathrm{J}$ stent, nephrostomy tube diameter and stay duration. These data were compared for postoperative urine leak.

Results: Urinary leakage was revealed in 76 of 108 patients (70.3\%). There was no statistically significant relationship between individual factors and urinary leakage $(\mathrm{P}>0.05)$. Among renal factors, just parenchymal cortical thickness and grade of hydronephrosis were strongly related to the urinary leakage $(\mathrm{P}<0.001)$. The renal stone burden did not affect urinary leakage $(\mathrm{P}$ $>0.05$ ). Among surgical factors, placing of ureteral double-J stent highly minimized the incidence of urinary leakage $(\mathrm{P}<0.001)$, and the urinary leakage significantly correlated with the increased diameter of the nephrostomy tube and staying duration $(\mathrm{P}<0.05)$.

Conclusion: After PCNL surgery, some factors show to influence post-PCNL urinary leakage. however most of the urinary leakage was treated conservatively, but it increases morbidity and hospitalization.
\end{abstract}

Keywords: Percutaneous nephrolithotomy; postsurgical complications; risk-factor; leaking of urine

Corresponding Author: karwanasaad87@gmail.com

Received: $15^{\text {th }}$ August 2020

Accepted: $7^{\text {th }}$ September 2020

DOI:https://doi.org/10.26505/DJM.19025510815

${ }^{1,2}$ Rizgary Teaching Hospital - Erbil- Iraq
${ }^{3}$ College of Medicine- Hawler Medical University- Erbil- Iraq

\section{Introduction}

Percutaneous nephrolithotomy (PCNL) was urinary stone disease affects $5-15 \%$ of first performed in the early of 1971s. The people all over the world. Open surgery was 
the main surgical therapy but has now been replaced by less invasive techniques including ESWL, PCNL, and URS(ureterorenoscopy)[1]. PCNL is the removal of a kidney stone via a tract developed between the surface of the skin and the collecting system of the kidney. It is the preferred treatment for large renal stones more than $2.5 \mathrm{~cm}$, stones resistant to ESWL, and some upper ureteric stones[2]. Currently, PCNL remains the mainstay of treatment of all types of renal calculi with a high success rate and stone-free rate. The complication rate was notably low in the PCNL procedure compared with another procedure to treat any kind of renal stone. Thus, in the past few decades, nearly all open surgeries for treating renal stones whether simple stones or complex staghorn stones have been changed to this minimally invasive procedure[3]. Improvement in technique and instruments have diminished the complication rate associated with this procedure. However, complications such as hemorrhage encountered in 1- $23 \%$ of cases, intrathoracic complication, observed in 2-12.5\%, and other organ injuries, observed in less than $1 \%$, are being reported with percutaneous renal surgery[4]. After normal PCNL procedure, nephrostomy tubes of different diameters are used for drainage of kidney and tamponade bleeding[5]. Traditionally, a nephrostomy catheter is usually inserted for about 1 to 2 days following the finishing of PCNL for drainage of urine, hemostasis, and healing of the nephrostomy catheter tract site [6]. In the present study, we discussed the parameters affecting leakage of urine following standard PCNL.

\section{Patients and Methods}

A total of one hundred and eight (108) patients who underwent PCNL were carried out by many urosurgeons in the urology department at Rizgary teaching hospital and Zheen international hospital. From February 2019 to October 2019 which was involved in this prospective case-study. There were 47 female and 61 male patients with a median age of 48.2 years (range:18-76). The ethical approval of the present study was taken from the local health ethics committee in Erbil city. The written consent form was taken from all patients before recruitment into the study. The confidentiality of the personal information of the patients was protected throughout the study period.

The inclusion criteria were renal stones. Exclusion criteria were (urinary tract infection, age less than 18 years old, change to open" PCNL failure", post-PCNL residual stone fragment ).

All patients were well prepared and received intravenous antibiotics preoperatively and postoperatively, Then all PCNL operations done under general anesthesia in a lithotomy position for inserting retrograde internal double $\mathrm{J}$ stent then shift to the prone position. The first percutaneous puncture to the choose calyx was performed under the fluoroscopy guide by using an 18 gauge needle after the installation of a contrast study (retrograde pyelogram). After puncturing the pelvicalyceal system with a guidewire, dilatation was adjusted with dilators (Ampltaz). Despite the procedure, dilations finished with the placing of a $28 \mathrm{~F}$ Amplatz sheath and through the sheath, 26F rigid nephroscope was placed. At the finishing of 
the surgery, the pelvicalyceal system and ureter were observed closely under direct vision by nephroscope. The decision for double J Stent placement was based on the suspect of presence residual small stones, prolonged renal manipulations, and association with hematuria. The decision for removing of nephrostomy tube was done according to the urine color in the nephrostomy tube, the volume of urine output, and the status of pain associated with the nephrostomy catheter. The patients can be discharged after 24-hours of hospitalization after removing the nephrostomy tube.

Patients were divided into 4 categories groups. The first one, individual factors composed of gender, body mass index (BMI), and age. The second one, renal factors composed of a previous operation (open or percutaneous surgery), history of ESWL, parenchymal cortical thickness, and the grade of hydronephrosis was detected by CT contrast or ultrasonography. The third one, Renal stone burden was measured by square area in millimeters $(\mathrm{mm})$ by multiplying the length(l) and the width(w) obtained before operation by KUB radiography. The fourth one, Surgical factors included the number of tracts, size of nephrostomy tube drainage, and duration of nephrostomy tube drainage, the existence of DJ stent. Patients who promptly become dry or leaking of urine stopped less than 12 hours after nephrostomy tube removal was calculated in the same group of "no leak".because this urine leakage stopped within 12-hours does not cause more hospitalization which permitted to discharge within 24-hours. The existence of leaking was defined by wetted dressing either notified by the patient or nurses or doctors.

\section{Statistical analysis}

Data were summarized and analyzed by using the Statistical Package for Social Sciences (SPSS-version 20). For the leaking of urine, a Chi-Square test and Student's ttest were used. A P-value of less than 0.05 was considered statistically significant.

\section{Results}

Leaking of urine was revealed in 76 of 108 cases $(70.3 \%)$. The allocation of patients according to the presence and absence of Leaking urine depending on the individual factor, Kidney factors, and surgical factors, in Tables $(1,2)$ and $(3)$, are shown. Also, those tables summed up the statistical significance of the presence of urinary leakage (PUL) regarding all defined parameters .

There was no significant relationship between individual factors (age, gender, $\mathrm{BMI}$ ) and the presence of urinary leakage $(\mathrm{P}>0.05)$. Body mass index data were taken randomly from the patients. 
Table (1): Individual factors according to postoperative urinary leakage

\begin{tabular}{|c|c|c|c|}
\hline & \multicolumn{2}{|c|}{ Urinary leakage (No.) } & \multirow[b]{2}{*}{ P-value } \\
\hline & Absent & Present & \\
\hline \multicolumn{4}{|l|}{ Sex (No.) } \\
\hline Male & 19 & 42 & \multirow{2}{*}{0.622} \\
\hline Female & 13 & 34 & \\
\hline Total & 32 & 76 & \\
\hline \multicolumn{4}{|l|}{ Age (year) } \\
\hline (No.) & 32 & 76 & \multirow[t]{2}{*}{0.774} \\
\hline Mean +/- SD & $48.3+/-15.6$ & $48.1+/-15.5$ & \\
\hline \multicolumn{4}{|c|}{ Body mass index } \\
\hline (No.) & 11 & 32 & \multirow[t]{2}{*}{0.722} \\
\hline Mean +/- SD & $27.8+/-5.44$ & $28.5+/-4.85$ & \\
\hline
\end{tabular}

No major difference was noted in the presence of urine leakage concerning the presence of prior operations or history of ESWL ( $\mathrm{P}>0.05)$ statistically non-significant. Among renal factors, only the parenchymal cortical thickness and grade of hydronephrosis were significant with the presence of urine leakage $(\mathrm{P}<0.001)$.

About the nephrostomy catheter, the average size was 20 Fr (range:14-24), and they were placed for a mean time of 36 hours postoperatively. if the nephrostomy tubes remained more than 36 hours postoperatively (increased time to catheter removal) will increase the incidence of the urinary leakage $(\mathrm{P}<0.05)$, double $\mathrm{J}$ stent significantly decreased the presence of urine leakage $(\mathrm{P}<0.05)$ than stentless. Urinary leaking was significantly elevated by increasing the nephrostomy tube diameter $(\mathrm{P}$ $<0.05)$.

Table (2): Renal factors according to the state of urine leakage

\begin{tabular}{|c|c|c|c|c|c|}
\hline & \multicolumn{4}{|c|}{ Urinary leakage (No.) } & \multirow{3}{*}{ P-value } \\
\hline & \multicolumn{2}{|c|}{ Absent } & \multicolumn{2}{|c|}{ Present } & \\
\hline & No. & $(\%)$ & No. & $(\%)$ & \\
\hline \multicolumn{6}{|l|}{ History of ESWL } \\
\hline Negative & 24 & $(28.6 \%)$ & 60 & (71.4\%) & \multirow{3}{*}{0.449} \\
\hline Posetive & 8 & $(33 \%)$ & 16 & $(67 \%)$ & \\
\hline Total & \multicolumn{2}{|c|}{32} & \multicolumn{2}{|c|}{76} & \\
\hline \multicolumn{6}{|l|}{ Operation history } \\
\hline Negative & 26 & $(31 \%)$ & 58 & $(69 \%)$ & \multirow{3}{*}{0.626} \\
\hline Posetive & 6 & $(25 \%)$ & 18 & $(75 \%)$ & \\
\hline Total & \multicolumn{2}{|c|}{32} & \multicolumn{2}{|c|}{76} & \\
\hline \multicolumn{6}{|l|}{ PCT } \\
\hline$\geq 15 \mathrm{~mm}$ & 10 & $(45.5 \%)$ & 12 & $(54.5 \%)$ & \multirow{3}{*}{$<0.001$} \\
\hline$<15 \mathrm{~mm}$ & 22 & $(25.5 \%)$ & 64 & $(74.5 \%)$ & \\
\hline Total & \multicolumn{2}{|c|}{32} & \multicolumn{2}{|c|}{76} & \\
\hline $\begin{array}{c}\text { Grade of } \\
\text { Hydronephrosis }\end{array}$ & & & & & \\
\hline
\end{tabular}




\begin{tabular}{|c||c||c||c||c||}
\hline 0 & 10 & $(45.5 \%)$ & 12 & $(54.5 \%)$ \\
\hline 1 & 11 & $(27 \%)$ & 30 & $(73 \%)$ \\
\hline 2 & 9 & $(27 \%)$ & 26 & $(73 \%)$ \\
0
\end{tabular}

* ESWL (extracorporeal shockwave lithotripsy), PCT ( parenchymal cortical thickness )

The mean renal stone burden was $403 \mathrm{~mm}^{2}$ (range: $28-2,600 \mathrm{~mm}^{2}$ ) in the non-leakage group and $635 \mathrm{~mm}^{2}$ (range $37-3,900 \mathrm{~mm}^{2}$ ) in the positive leakage group. The median stone burden did not impact on the leaking of urine $(\mathrm{P}=0.826)$.

Table (3): Surgical factors associated with urinary leakage

\begin{tabular}{|c|c|c|c|c|c|}
\hline & \multicolumn{4}{|c|}{ Urinary leakage (No.) } & \multirow{3}{*}{ P-valuc } \\
\hline & \multicolumn{2}{|c|}{ Absent } & \multicolumn{2}{|c|}{ Present } & \\
\hline & No. & $(\%)$ & No. & $(\%)$ & \\
\hline \multicolumn{6}{|c|}{ Tract number } \\
\hline 1 & 21 & $(27 \%)$ & 56 & (73\%) & \multirow{3}{*}{0.237} \\
\hline$>1$ & 11 & $(35 \%)$ & 20 & $(65 \%)$ & \\
\hline Total & \multicolumn{2}{|c|}{32} & \multicolumn{2}{|r|}{76} & \\
\hline \multicolumn{6}{|l|}{ J S Stent use } \\
\hline Absent & 6 & $(12.5 \%)$ & 42 & $(87.5 \%)$ & \multirow{3}{*}{$<0.001$} \\
\hline Present & 26 & $(43.3 \%)$ & 34 & $(56.7 \%)$ & \\
\hline Total & \multicolumn{2}{|c|}{32} & \multicolumn{2}{|c|}{76} & \\
\hline \multicolumn{6}{|c|}{$\begin{array}{l}\text { PCN tube diameter } \\
\text { Fr }\end{array}$} \\
\hline$<18 \mathrm{Fr}$ & 24 & $(47 \%)$ & 27 & $(53 \%)$ & \multirow[b]{2}{*}{$<0.001$} \\
\hline $18-24 \mathrm{Fr}$ & 8 & $(14 \%)$ & 49 & $(86 \%)$ & \\
\hline Total & \multicolumn{2}{|c|}{32} & \multicolumn{2}{|r|}{76} & \\
\hline \multicolumn{6}{|c|}{$\begin{array}{l}\text { PCN tube stay } \\
\text { duration }\end{array}$} \\
\hline$<36$ hours & 17 & $(41 \%)$ & 24 & $(59 \%)$ & \multirow{3}{*}{0.001} \\
\hline$\geq 36$ hours & 15 & $(22 \%)$ & 52 & $(78 \%)$ & \\
\hline Total & \multicolumn{2}{|c|}{32} & \multicolumn{2}{|r|}{76} & \\
\hline
\end{tabular}

\section{Discussion}

Percutaneous nephrolithotomy has been the best method to treat stones $>2 \mathrm{~cm}$, staghorn stones, and stones of lower pole $>1 \mathrm{~cm}$. In addition to the more successful rate, a low morbidity ratio makes it more beneficial than other surgical procedures $[7,8]$.

A nephrostomy catheter is inserted into the nephrostomy tract after clearing the stone from calyces during the operation. This catheter improves hemostasis and prevents extravasation of urine, thus, preventing the formation of urinoma. Whatever urine leakage from the drain site after removal of the postoperative nephrostomy catheter is a common problem. Conventionally, 20-22 Fr nephrostomy catheter drainage has been preferred after finishing PCNL to provide good urinary drainage, prevent blood loss by pressuring the percutaneous nephrostomy tract, and maintaining a channel for the next interventions if needed. Nephrostomy catheters have been a problem in causing 
post-surgery pain and morbidity such as urinary leakage and blood loss[9]. The size of the nephrostomy catheter may also be associated with post-operative discomfort [12]. As discussed by Lee et al., PCNL involves many complications such as bleeding may need a blood transfusion, leakage of urine, urinary tract infection (UTI), fever, prolonged leakage of urine from the flank in fifty percent of the patients[10]. Urinary leakage, urinoma, and serious complications such as adjacent organ injury, urosepsis, and associated diseases like renal impairment, high blood sugar, obesity, metabolic syndromes, or cardiovascular illness increase perioperative complications [11].

Faerber and Goh et al. have noted that usually more complication was noted in obese patients than those patients who have normal BMI undergoing PCNL[15]. Dirim et al. have reported that individual factors (BMI, gender, age) did not affect the presence of urinary leakage[16]. Ansari et al.concluded that age, gender, and BMI did not influence postoperative complications like urinary leakage [13]. Our present study findings are consistent that body mass index, age, and gender did not affect the leaking of urine after PCNL.

Other studies have determined that PCNL can be done safely in patients with a previous history of ESWL or open renal surgeries without increased complication rates and with success rates the same as that of PCNL in patients without prior open renal surgeries[17] [24]. Our present findings improved too that the previous operation or
ESWL treatment did not affect leakage of urine after PCNL.

Uyeturk et al. evaluated the relationship between the degree of renal hydronephrosis, renal parenchymal cortical thickness, and urinary leakage. That study concluded that urinary leakage increases in line with the degree of hydronephrosis and decreasing cortical thickness [18]. In the present study, both decreasing parenchymal cortical thickness and increasing the grades of hydronephrosis were shown to be highly related to urinary leakage, due to the effect on cortical thickness (hydronephrosis makes thinning of cortical parenchymal thickness). Each grade increasing at the grades of hydronephrosis caused three times increasing in tract site leaking of urine.

Turna et al, also have determined that the complications associated with maximized stone size and surface area[19]. Binbay et al. have determined the stone size was bigger in those patients group with prolongation of urinary leakage, and have improved that stone burden is predictive for prolongation of urinary leakage[21]. While in our study, whatever there was a non-significant correlation between stone burden and leakage of urine $(\mathrm{P}>0.05)$.

Desai et al. have demonstrated that prolongation of urinary leakage occurred $4.5 \%$ in single tract and $2.9 \%$ in multiple tract PCNL, the tract number has not correlated with post PCNL tract site urinary leakage [20]. Binbay et al. were determined that the number of tracts was not an effective factor for prolongation of urinary leak development [21]. In the present study, results have approved that the percutaneous 
tract number was not a significant factor for the development of prolonged urine leakage. Maheshwari et al. demonstrated that when a larger bore nephrostomy catheter is used, the Prolongation of urinary leakage becomes apparent in most patients after tube removal [22].

In other prospective randomized studies, Desai et al. were compared post-surgical outcomes between traditional small-bore ( 9 Fr) nephrostomy and large bore (20 Fr) nephrostomy drainage after PCNL. They reported the presence of the leaking urine for large bore much more than smaller bore groups respectively[23]. In our study is the same as the presence of leaking urine increased with increasing nephrostomy tube diameter and increasing the duration of tube removal $(\mathrm{P}<0.05)$. Using smaller bore tubes and early removal appear helpful to decrease the leaking of urine following nephrostomy catheter removal after PCNL.

Telha et al have determined that prolonged urinary leakage occurred 5.6\% in PCNL with the double-J stent and $17.1 \%$ in PCNL with the ureteric catheter, so the best method is double-J stent to decrease complications and urinary leakage[14]. Dirim et al demonstrated that using of double-J stent significantly reduced the incidence of urinary leakage after PCNL[16]. In the present study, utilizing the double $\mathrm{J}$ stent was decreased the incidence and presence of urinary leakage $(\mathrm{P}<0.001)$. Those patients, whom a Double J-stent has been inserted, must be informed about the complications of the double-J stent if not removed from the body at suitable times.

\section{Conclusions}

In the present study, several factors appear to influence post-PCNL urinary leakage. however most of the urinary leakage was treated conservatively, but it increases morbidity and hospitalization. so we advised those patients who are at risk of urinary leakage should use smaller diameter, shortduration nephrostomy catheter, and double $\mathrm{J}$ Stent should be placed at the end of PCNL surgery.

\section{Recommendations}

1.To prevent urinary tract infection and urosepsis, all patients should receive intravenous antibiotics preoperatively as prophylaxis.

2.We suggest that for patients who are at increased risk of prolonged urinary leakage, the double-J stent should be inserted at the end of the PCNL procedure.

3.More prospective studies are needed in the future to evaluate other factors like (UTI, post-PCNL residual stone fragments, chronic medical diseases, duration of operation, ..etc.) that affecting post PCNL procedure urinary leakage.

\section{References}

[1]Kim BS. Recent advancement or less invasive treatment of percutaneous nephrolithotomy. Korean J Urol. 2015;56(9):614-23.

[2]Takazawa R, Kitayama S, Tsujii $T$. Appropriate kidney stone size for ureteroscopic lithotripsy: When to switch to a percutaneous approach. World J Nephrol.2015; 4(1);11-7.

[3]Diri A, Diri B. Management of staghorn renal stones.Ren Fail. 2018; 40(1):357-62. 
[4]Davidoff R, Bellman GC. Influence of technique of percutaneous tract creation on incidence of renal hemorrhage. $\mathrm{J}$ Urol. 1997;157(4):1229-31.

[5]Labate G, Modi P, Timoney A, et al. The percutaneous nephrolithotomy global study: Classification of complications. J Endourol. 2011;25:1275-80.

[6] Yu DS. Gelatin packing of intracortical tract after percutaneous nephrostomy lithotripsy for decreasing bleeding and urine leakage. J Chin Med Assoc 2006;69:162165.

[7]Srivastava A, Chipde SS. Management of $1-2 \mathrm{~cm}$ renal stones. Indian journal of urology: IJU: journal of the Urological Society of India. 2013 Jul;29(3):195.

[8]Assimos D, Krambeck A, Miller NL, et al. Guideline Statement. J Urol. 2016 Oct;196(4):1153-60.

[9]Tefekli A, Altunrende F, Tepeler K, Tas A, Aydin S, Muslumanoglu AY.Tubeless percutaneous nephrolithotomy in selected patients: a prospective randomized comparison. Int Urol Nephrol 2006;39:5763.

[10]Lee WJ, Smith AD, Cubelli V, et al. Complications of percutaneous nephrolithotomy. AJR Am J Roentgenol 1987;148:177-180.

[11]Taylor E, Miller J, Chi T, Stoller ML. Complications associated with percutaneous nephrolithotomy. Transl Androl Urol. 2012;1(4):223-228.

[12]Weiland D, Pedro RN, Anderson JK, et al. Randomized prospective evaluation of nephrostomy tube configuration: impact on postoperative pain. International braz j urol. 2007 Jun;33(3):313-22.
[13] Ansari H, Tomar V, Yadav SS, Agarwal N. Study of predictive factors affecting the prolonged urinary leakage after percutaneous nephrolithotomy. Urology annals. 2016 Jan;8(1):60.

[14]Telha KA, Alba'adani TH, Alkohlany KM, Al-Adimy AO, Alnono IH. Tubeless percutaneous nephrolithotomy with double-J stent compared with external ureteral catheter to decrease postoperative complications. Saudi Med. J. 2010 Oct;31(10):1137-40.

[15] Faerber GJ, Goh M (1997) Percutaneous nephrolithotripsy in the morbidly obese patient. Tech Urol. 1997;3(2):89-95.

[16]Dirim A, Turunc T, Kuzgunbay B, Hasirci E, Tekin MI, Ozkardes H. Which factors may effect urinary leakage following percutaneous nephrolithotomy?. World J. Urol. 2011 Dec 1;29(6):761-6.

[17]Resorlu B, Kara C, Senocak C, Cicekbilek I, Unsal A. Effect of previous open renal surgery and failed extracorporeal shockwave lithotripsy on the performance and outcomes of percutaneous nephrolithotomy. J Endourol. 2010;24(1):136.

[18]Uyeturk U, Gucuk A, Kemahli E, et al. Factors influencing the duration of urine leakage following percutaneous nephrolithotomy. Advances in Urology. 2014 Feb 4;2014.

[19]Turna B, Umul M, Demiryoguran S, Altay B, Nazli O, How do increasing stone surface area and stone configuration affect overall outcome of percutaneous nephrolithotomy? J Endourol. 2007;21(1):34-43. [20]Desai MR, Kukreja RA, Patel SH, Bapat SD. Percutaneous nephrolithotomy for 
complex pediatric renal calculus disease. J Endourol. 2004 Feb 1;18(1):23-7.

[21]Binbay M, Sari E, Tepeler A, et al. Characteristics of patients requiring Double-J placement because of urine leakage after percutaneous nephrolithotomy. J Endourol. 2009 Dec 1;23(12):1945-50.

[22]Maheshwari Pn, Andankar Mg, Bansal M. Nephrostomy tube after percutaneous nephrolithotomy: large-bore or pigtail catheter? J Endourol. 2000 Nov;14(9):735-8. [23]Desai MR, Kukreja RA, Desai MM, et al. A prospective randomized comparison of type of nephrostomy drainage following percutaneous nephrostolithotomy: large bore versus small bore versus tubeless. J Urol. 2004;172:565-567.

[24]Tugcu V, Su FE, Kalfazade N, Sahin S, Ozbay B, Tasci AI. Percutaneous nephrolithotomy (PCNL) in patients with previous open stone surgery. Int Urol Nephrol. 2008 Dec 1;40(4):881-4. 